Review

\title{
Diagnosis Accuracy and Prognostic Significance of the Dickkopf-1 Protein in Gastrointestinal Carcinomas: Systematic Review and Network Meta-analysis
}

Xiaowen Jiang1\#, Fuhai Hui1" ${ }^{1 \#}$ Xiaochun Qin ${ }^{1}$, Yuting $\mathrm{Wu}^{1}$, Haihan $\mathrm{Liu}^{1}$, Jing Gao², Xiang $\mathrm{Li}^{2}$, Yali $\mathrm{Xu}^{2}$, Yingshi Zhang ${ }^{1 凶}$

1. Department of Life Science and Biochemistry, Shenyang Pharmaceutical University, Shenyang, 110016, China

2. Department of Pharmacy, Shenyang Pharmaceutical University, Shenyang, 110016, China.

\#Xiaowen Jiang and Fuhai Hui contributed equally to this article

$\triangle$ Corresponding author: Yingshi Zhang, Department of Life Science and Biochemistry, Shenyang Pharmaceutical University, No. 103 Wenhua Road, Shenyang, 110016, China. E-mail: 104040309@syphu.edu.cn, zhangyingshi526@163.com (Y. Zhang)

(C) The author(s). This is an open access article distributed under the terms of the Creative Commons Attribution License (https://creativecommons.org/licenses/by/4.0/). See http://ivyspring.com/terms for full terms and conditions.

Received: 2020.06.26; Accepted: 2020.10.06; Published: 2020.10.18

\begin{abstract}
Objective: To evaluate the diagnosis accuracy and prognostic significance of bio-marker dickkopf-1(DKK-1) protein in GIC, and also sub-type of hepatocellular carcinoma (HCC), pancreas carcinomas (PC), oesophageal carcinoma (EPC) and Adenocarcinoma of esophago-gastric junction (AEGJ), etc.
\end{abstract}

Methods: Electronic databases were searched from inception to May 2020. Patients were diagnosed with gastrointestinal carcinomas, and provided data on the correlation between high and low DKK-1 expression and diagnosis or prognosis.

Results: Forty-three publications involving 9318 participants were included in the network meta-analysis, with 31 of them providing data for diagnosis value and 18 records were eligible for providing prognosis value of DKK-1. DKK-1 has a moderate diagnostic value for overall GIC, $\mathrm{HCC}$ and PC. In addition, for the combined diagnosis value of DKK-1 +AFP, high diagnostic accuracy value could be determined in $\mathrm{HCC}$ and early HCC group, respectively. Whereas, diagnosis efficiency of DKK-1+CA19-9 was also better than that of DKK-1 alone with $A U C$ value is above 0.95 . For the prognosis meta-analysis of histopathological stratification, we found that EPC and AEGJ ranked the best for the histopathological stratification of prognosis from network meta-analysis. This systematic review protocol was registered with the PROSPERO registry (No.CRD42020167910).

Conclusion: DKK-1 has good diagnostic accuracy, especially combination of DKK-1+AFP in HCC and DKK-1+CA19-9 in PC, whereas modest prognostic significant in GIC. Future head-to-head researches are warranted for DKK-1 expression in HCC and PC tissue.

Key words: DKK-1; Gastrointestinal carcinomas; Diagnosis; Prognostic; Network meta-analysis

\section{Introduction}

Gastrointestinal carcinomas (GIC), mainly including liver carcinoma (LC), pancreatic carcinoma (PC), gastric carcinomas (GC), oesophageal carcinoma (EPC) and colorectal carcinoma (CRC), are the causes of high morbidity and mortality worldwide[1]. In addition, GIC represents a significant health burden in society, and its prevalence is inclined to continue to grow in the future[2]. LC contains two types of histopathology, hepatocellular carcinoma (HCC) and intrahepatic cholangiocarcinoma (IHCC), with a proportion of HCC is greater than 95\% [3]. GIC has the characteristics of a high degree of differentiation, concealed early onset, rapid development in the middle stage, and easy metastasis and recurrence in 
the late stage; thus, most patients are in the middle and late stages when clinically diagnosed[4]. Therefore, it is extremely important to find a biomarker with better diagnostic value to diagnose GIC early, as it is very important to improve the overall survival of GIC patients. It is expected to have a better predictive effect of histopathological stratification to predict the evolution and prognosis of patients' condition, which is of great significance for the overall control of patients' evolution and prognosis changes[5-6].

Dickkopf-1 (DKK-1), a secreted glycoprotein that acts as an antagonist of the Wnt/ $\beta$-catenin signalling pathway, is part of the DKK family of proteins that includes Dkk-2, Dkk-3 and Dkk-4. The Wnt/ $\beta$-catenin signalling cascade governs cell proliferation and cell fate during embryonic development and tissue homeostasis[7-8]. The Wnt/ $\beta$-catenin signalling pathway is one of the most important pathways in the initiation and progression of GIC[9-10]. Currently, the biomarker DKK-1 is being studied and is highly expressed in various GIC[11-12]. However, its diagnostic and prognostic efficacy for the most suitable subtype of GIC has not been determined. Even if there were previously published meta-analyses, the included original articles were not comprehensive enough, and no previous systematic review has provided a comprehensive overview with meta-regression and network meta-analysis for diagnostic and prognostic data.

\section{Methods}

The network meta-analysis of this systematic review was structured according to the PRISMA (preferred reporting items for systematic reviews and meta-analyses) statement for diagnostic test accuracy and prognosis test significance[13-14]. This systematic review protocol was registered with the PROSPERO registry (No. CRD42020167910)[15].

\section{Search Strategy and Selection Criteria}

Four electronic databases (PubMed, Embase, the Cochrane Library and China National Knowledge Infrastructure) were searched using "Dickkopf-1", "DKK-1", "digestive", "alimentary", "gastrointestinal", "cancer", "carcinoma", and "neoplasms" and their MeSH terms from inception to April 2020 (details are provided in Table S1 in the Supplement). Two researchers (L.H.Y. and H.F.H.) independently screened the titles and abstracts of original publications identified through the electronic search or reviewed the full text of potentially relevant articles as needed to retrieve the eligibility for inclusion in this systematic review and meta-analysis. Records published in the English or Chinese were included if they were described as retrospective and prospective observational studies. Any discrepancies were resolved by consensus with two experienced researchers (Z.Q.C. and Z.Y.S.). Studies were included if they met the following criteria: the original research type was diagnostic or prognostic. Patients were diagnosed with gastrointestinal carcinomas (including HCC, PC, GC, EPC, etc.), and provided data on the correlation between high and low DKK-1 expression and diagnosis or prognosis. Case reports, case series, studies without human data, and conference abstracts were excluded.

\section{Data Extraction and Quality Assessment}

All data extraction from each study was independently undertaken by two researchers (J.X.W. and H.F.H.) using predesigned forms. For diagnostic-type research, basic characteristics (first author, publication year, country, cancer type, control type, case count, and control count), clinical features (biomarker test method, DKK-1 cut-off values, treatment, and all biomarkers used) and a $2 \times 2$ data table of true positive, false negative, true negative, and false positive results were used. For prognostic-type research, basic characteristics (first author, publication year, country, high/low expression count, cancer type, and control type), clinical features (patient treatment, research type, biomarker test method, DKK-1 cut-off values, and all biomarkers used) and histopathological features (tumour size, TNM stage, differentiation grade, lymphatic invasion, lymph node metastasis, vascular invasion, and distant metastasis) were assessed.

We used a modified Quality Assessment of Diagnostic Accuracy Studies 2 (QUADAS-2) tool to assess the quality of DKK-1 as one of the biomarker discovery studies[16]. QUADAS-2 consists of 4 domains, including patient selection, index test, reference standard, and flow of patients through the study, which could be included without high-risk options. We also used the Newcastle-Ottawa Scale (NOS) scale[17] for observational study, which is used to determine the quality of DKK-1 as one of the prognostic indicators. NOS scores greater than 4 (max 10) can be included in this meta-analysis, and study quality was also independently assessed by two independent researchers.

\section{Outcomes and Analysis}

For diagnostic-type research, high expression of DKK-1 alone or in combination with other biomarkers (DKK-1+AFP, DKK-1+CA19-9) was the indicator under investigation, and the sensitivity, specificity, positive likelihood ratio (PLR), negative likelihood ratio (NLR), diagnostic odds ratio (DOR) and the area 
under the receiver operating characteristic (ROC) curves were derived from diagnostic models of overall participants and subgroup studies for each type of carcinoma, including HCC, early HCC, PC, early PC, GC, EPC.

For prognostic-type research, high expression of DKK-1 alone) was the indicator under investigation. Histopathological stratification included tumour size $(>5 \mathrm{~cm}$ vs $\leq 5 \mathrm{~cm})$, TNM stage (III-IV vs I-II), differentiation grade (poor vs well/moderate), lymphatic invasion (yes/no), lymph node metastasis (yes/no), vascular invasion (yes/no) and distant metastasis (yes/no), which could also be subgrouped by cancer type (HCC, PC, GC, and EPC) and test method (IHC and ELISA).

\section{Synthesis of Evidence}

To pool results from diagnostic-type research, we applied the hierarchical summary ROC model[18] and obtained summary point estimates of the pairs of sensitivity and specificity, as well as DOR, PLR and NLR with their 95\% confidence intervals (CIs). Summary estimates of the test accuracy were plotted in the ROC space together with the summary ROC curve[19]. To estimate the results from prognostic-type research, pooled odds ratios (ORs) or standardized mean differences (SMDs) with their 95\% CIs were used to obtain a summary of the significant differences. The $I^{2}$ statistic was used to assess the statistical heterogeneity among the included studies, when $I^{2}>50 \%$ indicated high heterogeneity[20], and regardless of heterogeneous results, random effects models were applied[21]. Subgroup analyses and meta-regression were performed on the basis of cancer type and test method. A $P$ value less than 0.05 from the meta-regression indicated that this grouping method had a great impact on the overall results. If the network meta-analysis was demanded, the surface under the cumulative ranking (SUCRA) probabilities were used to rank them, and the higher SUCRA scores corresponded to a greater efficacy. Potential publication bias was evaluated by Deeks' asymmetry test for diagnostic-type outcomes and Harbord's test for prognostic-type outcomes[22]. In this study, MetaDisc (version 1.4) and STATAMP (version 14.0) software were used.

In addition, the quality of evidence for the diagnostic-type outcomes and prognostic-type research was assessed based on the GRADE system to estimate grading of recommendations, assessments, developments, and evaluations for diagnostic-type outcomes[23], risk of bias (study limitations), imprecision, inconsistency, indirectness of study results, and publication bias for prognostic-type outcomes[24].

\section{Results}

\section{Study characteristics and Quality assessment}

From 784 potential records identified through PubMed, Embase, the Cochrane Library, and China National Knowledge Infrastructure (CNKI), the literature systemic search yielded 43 publications [25-67], including 9318 participants who met the eligibility criteria; of these participants, 31 of them had diagnostic data for DKK-1, and 18 records provided prognostic data for DKK-1(Figure 1). The number of subjects with or without GIC included in those publications ranged from 31 to 831. Among all included studies, HCC $(n=19)$, PC $(n=6)$, GC $(n=9)$, EPC $(\mathrm{n}=6), \quad \mathrm{CRC} \quad(\mathrm{n}=1)$, intrahepatic cholangiocarcinoma (IHCC, $n=1)$ and adenocarcinoma of the oesophagogastric junction (AEGJ, n=1) were researched. For the detection method of the DKK-1 protein, 31 publications utilized the enzyme-linked immunosorbent assay (ELISA) to detect the expression of DKK-1, and the other 12 articles used the immunohistochemical (IHC) method (Table 1 and Table S2 in the Supplement). Moreover, many of the studies we included not only used BKK-1 as a tumour biomarker but also explored the diagnostic synergistic effects of DKK-1 and other markers, including the combination of AFP in HCC and the combination of CA19-9 in PC. In addition, the results from baseline pairwise analysis showed that among the diagnostic tests, the GIC patient group showed a higher age and more males (Table $\mathbf{1}$ ). Quality assessments for the original diagnostic research by QUADAS-2 scales are summarized in Table S3; the original prognostic research by CASP scales are summarized in Table S4. All of our included publications had acceptable quality.

\section{Results of the combined diagnostic value of DKK-1}

We first meta-analysed data for the diagnostic value of DKK-1 in overall GIC. The sensitivity and specificity were 0.70 (95\% CI: $0.69-0.71)$ and 0.82 (0.81-0.83), respectively, with an AUC score of 0.8365, which means that the diagnostic value was moderate (Table 2). Therefore, we performed subgroup analysis and meta-regression to determine which type of GIC was most suitable for diagnosing DKK-1. No significant differences were found from the meta-regression $(P=0.06)$ among different GIC. Of the eligible studies, 17 studies reported data on overall HCC, and the diagnostic value was also moderate, with the sub-subgroup study in the HCC group. When the control group was patients with $\mathrm{LC} \pm \mathrm{HBV} \pm \mathrm{HCV}$ from 7 studies, the diagnostic value was also moderate. When the intervention group was 
limited to patients with early HCC, the diagnostic value was also similar to that above. No significant results were found from the meta-regression in the above two sub-subgroups. For the diagnostic efficacy of DKK-1 in PC, the overall diagnostic value was higher, with an ROC score over 0.8818 . For patients limited to early PC, the number of included studies was too small to determine the diagnostic value. For subgroup GIC types focusing on EPC and GC, low diagnostic value was detected (Table 2).

For the estimation of DKK-1's diagnostic efficacy, its combined diagnostic effect also needs to be considered because if the combined diagnostic efficiency was better, it also indicates that the expression of DKK-1 was more meaningful. For the combined diagnostic efficacy of DKK-1, we considered the diagnostic value of DKK-1+AFP in patients with HCC to be high, with an AUC of 0.9211. This is the combined diagnostic value of DKK-1 and AFP, which can also be said to improve the diagnostic efficiency of AFP from AUC=0.7941 (Table S5). Subgroups of the control group were patients with $\mathrm{LC} \pm \mathrm{HBV} \pm \mathrm{HCV}$, and the diagnostic value was moderate. While the sub-subgroup of the intervention group was early $\mathrm{HCC}$, the diagnostic value of DKK-1+AFP was higher than that of the overall HCC group, with an AUC score of 0.9109. For the combination of the diagnostic value of DKK-1+CA19-9, the diagnostic value was higher than that of DKK-1 alone (0.9563), which was the highest of all diagnostic values. For the diagnostic value of DKK-1 alone or in combination, no publication bias was found among overall, subgroup and sub-subgroup outcomes with a low to high GRADE (Figure 2, Table 2). Generally speaking, the diagnostic value of DKK-1+AFP was high in the HCC and early HCC groups. Additionally, DKK-1 alone or in combination with CA19-9 was effective in diagnosing PC.

Potential records identified through initial searches of electronic databases $(n=784)$

Pubmed (n=395), Embase (n=297), Cochrane Library $(n=15)$, CNKI $(n=77)$

\begin{tabular}{|c|c|}
\hline & Duplicate records $(n=488)$ \\
\hline \multicolumn{2}{|c|}{ Title and abstract records assessed for eligibily $(n=296)$} \\
\hline & $\begin{array}{l}229 \text { records excluded } \\
\text { Reviews and meta-analysis }(n=86) \\
\text { No human studies }(n=31) \\
\text { The indicator is DKK3 or DKK-4 }(n=105) \\
\text { Outcome was survival rate }(n=7) \\
\end{array}$ \\
\hline \multicolumn{2}{|c|}{ Full-text studies evaluated for detail identified $(n=67)$} \\
\hline & $\begin{array}{l}24 \text { records excluded } \\
\text { No useful data }(n=20) \\
\text { Duplicate publications }(n=3) \\
\text { Single group study }(n=1)\end{array}$ \\
\hline \multicolumn{2}{|c|}{ Articles included quantitative synthesis and meta-analysis $(n=43)$} \\
\hline$\downarrow$ & $\downarrow$ \\
\hline $\begin{array}{l}31 \text { articles (52 analyses)evaluated } \\
\text { diagnostic value of DKK-1 in GIC } \\
17 \text { studies on HCC } \\
6 \text { studies on PC } \\
2 \text { studies on GC } \\
4 \text { studies on EPC } \\
2 \text { studies on other GIC }\end{array}$ & $\begin{array}{l}18 \text { articles (18 analyses) evaluated } \\
\text { prognostic value of DKK-1 in GIC } \\
3 \text { studies on HCC } \\
2 \text { studies on PC } \\
7 \text { studies on GC } \\
4 \text { studies on EPC } \\
2 \text { studies on other GIC }\end{array}$ \\
\hline
\end{tabular}

Figure 1. Flowchart summarizing publication search and study selection. 
Table 1. Main characteristics of the diagnostic and prognostic value of DKK-1 in GIC.

\begin{tabular}{|c|c|c|c|c|}
\hline Article type & $\begin{array}{l}\text { Cancer } \\
\text { type }\end{array}$ & Test method & No. of studies & $\begin{array}{l}\text { No. of } \\
\text { patients }\end{array}$ \\
\hline \multirow{9}{*}{$\begin{array}{l}\text { Diagnostic } \\
\text { articles }\end{array}$} & $\mathrm{HCC}$ & ELISA & 15 & 3962 \\
\hline & & $\mathrm{IHC}$ & 2 & 356 \\
\hline & PC & ELISA & 3 & 574 \\
\hline & & $\mathrm{IHC}$ & 3 & 94 \\
\hline & EPC & ELISA & 3 & 416 \\
\hline & & IHC & 1 & 288 \\
\hline & GC & ELISA & 2 & 267 \\
\hline & AEGJ & ELISA & 1 & 180 \\
\hline & CRC & ELISA & 1 & 385 \\
\hline \multirow{8}{*}{$\begin{array}{l}\text { Prognostic } \\
\text { articles }\end{array}$} & GC & IHC & 7 & 1328 \\
\hline & EPC & ELISA & 2 & 206 \\
\hline & & IHC & 2 & 220 \\
\hline & $\mathrm{HCC}$ & ELISA & 2 & 172 \\
\hline & & IHC & 1 & 75 \\
\hline & IHCC & IHC & 1 & 50 \\
\hline & PC & IHC & 2 & 355 \\
\hline & AEGJ & ELISA & 1 & 79 \\
\hline \multicolumn{2}{|c|}{ Baseline Characteristics } & Results & Heterogeneity & Significant \\
\hline \multirow[t]{2}{*}{ Diagnostic } & Sex & $1.40(1.17,1.67)$ & $0.071,29.3 \%$ & Yes \\
\hline & Age & $0.47(0.28,0.66)$ & $0.000,85.6 \%$ & Yes \\
\hline \multirow[t]{2}{*}{ Prognostic } & Sex & $1.02(0.76,1.37)$ & $0.286,16.1 \%$ & No \\
\hline & Age & $-0.09(-0.45,0.27)$ & $0.018,70.3 \%$ & No \\
\hline
\end{tabular}

AEGJ, adenocarcinoma of oesophagogastric junction; CRC, colorectal carcinoma; DKK-1, dickkopf-1; ELISA, enzyme-linked immunosorbent assay; EPC, oesophageal carcinoma; GIC, gastrointestinal carcinomas; HCC, hepatocellular carcinoma; IHC, immunohistochemistry; IHCC, intrahepatic cholangiocarcinoma; LC, liver carcinomas; PC, pancreatic carcinomas.

\section{Results of combined prognostic value of DKK-1}

Second, we performed subgroup meta-analysis and meta-regression for the histopathological stratification from subgroup analyses depending on the cancer type, and the test method used DKK-1 for the prognosis of GIC. For the outcome of tumour size, no significant differences were found in every subgroup; however, a low heterogeneity outcome was found in the GIC types of GC+EPC and GC alone. For the TNM meta-analysis, a significant difference was only found in the IHCC subgroup from only one study. This means that patients may have a more advanced TNM stage in the DKK-1 high expression group. For differentiation grade outcome, no significant differences could be found between high and low levels of DKK-1 expression. For the lymphatic invasion and lymph node metastasis groups, no significant results were found. When considering the vascular invasion results, no heterogeneity could be found in the overall LC group, the HCC group and the test method of the ELISA group, with no significant results, while no significant differences in distant metastasis outcome were found. No publication bias could be found in every group with low to moderate GRADE. In general, high DKK-1 expression may indicate a poor prognosis, especially in PC and IHCC. However, too few studies have been included in these two tumour subtypes, so we applied network meta-analysis to rank the analysis of tumour subtypes and test methods (Table 3).

To determine which was the most suitable subtype of GIC for the high expression of DKK-1 and which test method had a greater impact on the prognosis, we conducted a network meta-analysis for TNM stage and lymph node metastasis. For the combination of two outcomes, in EPC patients, high DKK-1 expression may be more related to histopathological stratification prognosis, but no significant difference exists (Figure 3, Table S6).

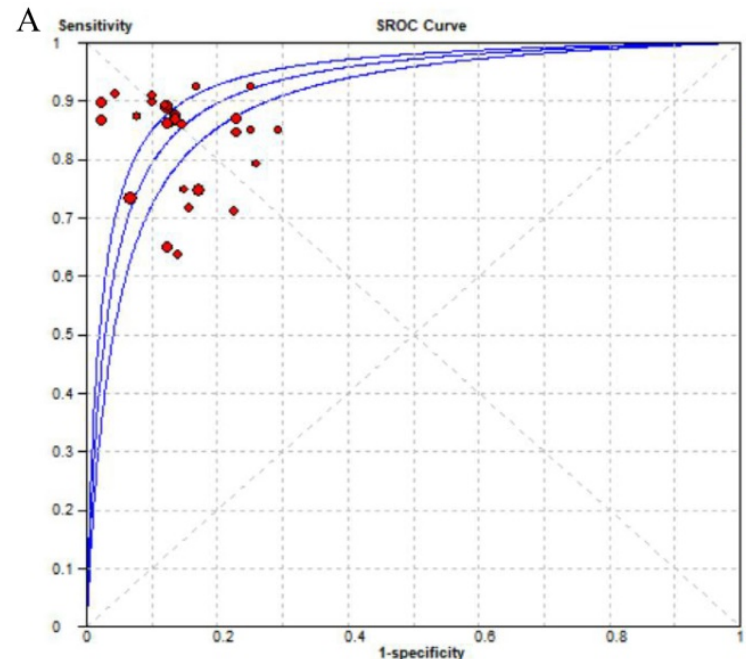

Diagnosis value of DKK-1+AFP in HCC
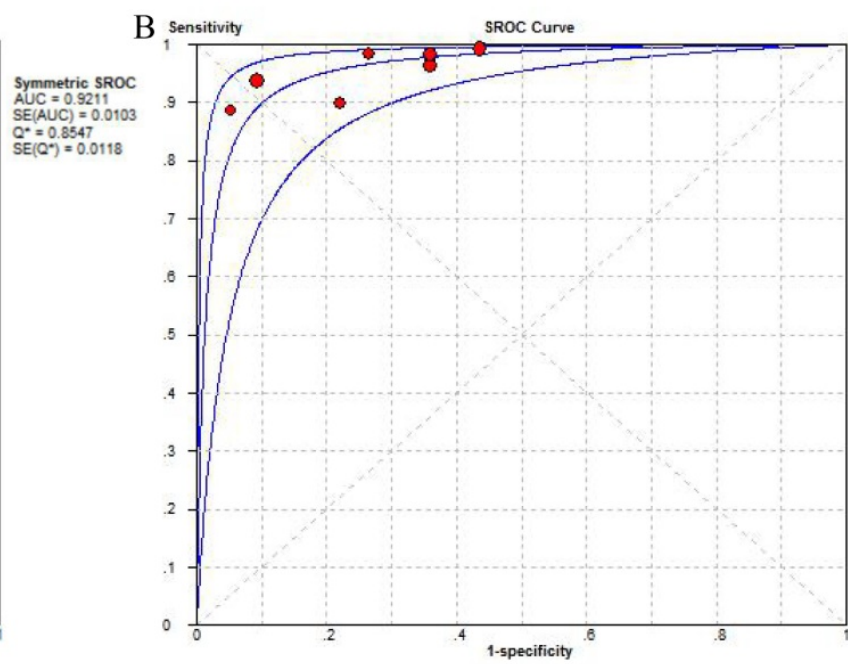

Diagnosis value of DKK-1+CA19-9 in PC
Symmetric SROC
$A \cup C=0.9563$ $\triangle E($ AUC $)=0.0168$ $\alpha^{*}=0.8992$

Figure 2. The pooled diagnostic accuracy of DKK-1+AFP in HCC diagnosis (A) and DKK-1+CA19-9 in PC diagnosis. DKK-1, dickkopf-1; HCC, hepatocellular carcinoma; PC, pancreatic carcinoma. 


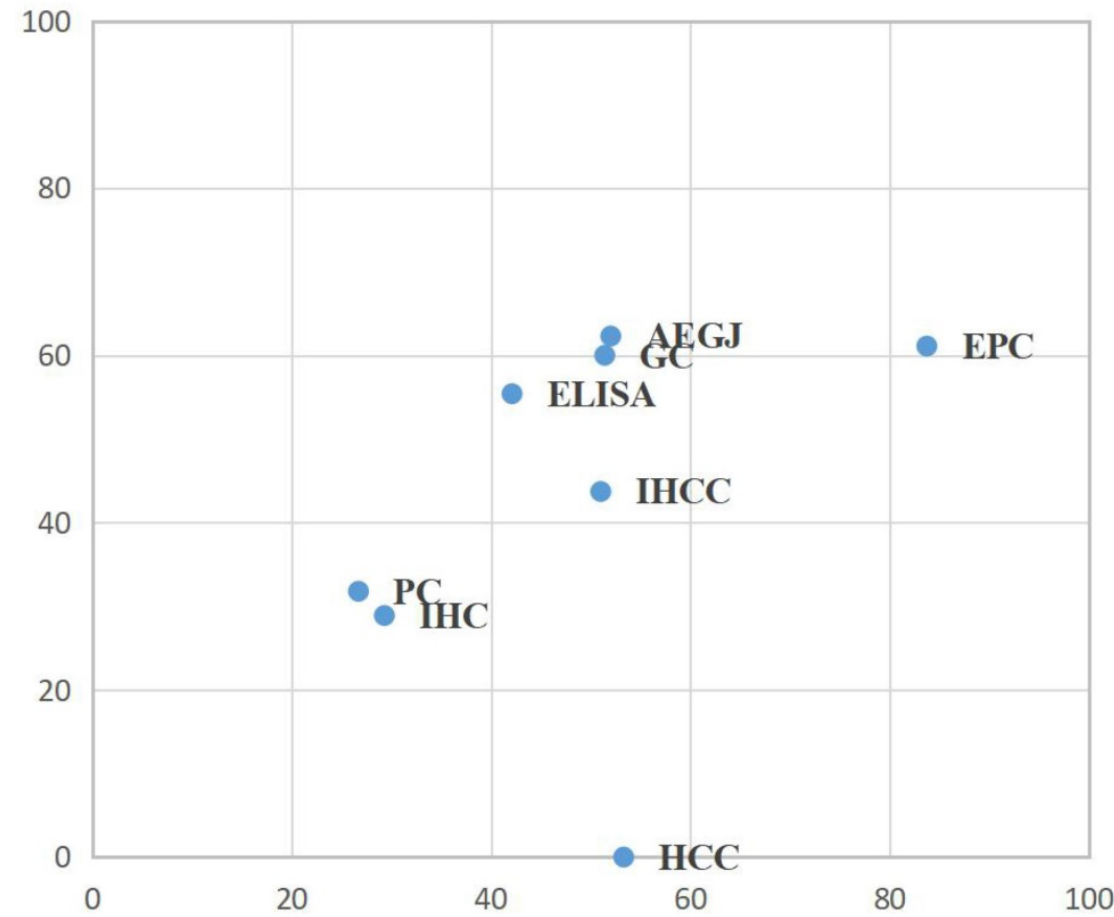

Figure 3. Surface under the cumulative ranking curve (SUCRA) rankings for TNM stage and lymph node metastasis in GIC patients. AEGJ, adenocarcinoma of oesophagogastric junction; CRC, colorectal carcinoma; DKK-1, dickkopf-1; ELISA, enzyme-linked immunosorbent assay; EPC, oesophageal carcinoma; GIC, gastrointestinal carcinomas; HCC, hepatocellular carcinoma; IHC, immunohistochemistry; IHCC, intrahepatic cholangiocarcinoma; LC, liver carcinomas; PC, pancreatic carcinomas.

Table 2. Summary estimates for the results from subgroup analyses depending on cancer type, cancer stage, control type used, and DKK-1 used alone or in combination for the diagnosis of GIC.

\begin{tabular}{|c|c|c|c|c|c|c|c|c|c|c|c|}
\hline & $\begin{array}{l}\text { No. of } \\
\text { Studies } \\
\text { (Analyses) }\end{array}$ & $\begin{array}{l}\text { No. of } \\
\text { participants } \\
\text { (there is } \\
\text { duplication) }\end{array}$ & $\begin{array}{l}\text { Sensitivity } \\
(95 \% \mathrm{CI})\end{array}$ & $\begin{array}{l}\text { Specificity } \\
(95 \% \text { CI })\end{array}$ & $\begin{array}{l}\text { Positive } \\
\text { Likelihood } \\
\text { Ratio (95\% } \\
\text { CI) }\end{array}$ & $\begin{array}{l}\text { Negative } \\
\text { Likelihood } \\
\text { Ratio (95\% } \\
\text { CI) }\end{array}$ & $\begin{array}{l}\text { Diagnostic } \\
\text { Odds } \\
\text { Ratio }(95 \% \\
\text { CI) }\end{array}$ & $\begin{array}{l}\text { Area } \\
\text { Under } \\
\text { the } \\
\text { Curve }\end{array}$ & Mete-regression & $\begin{array}{l}\text { Publication } \\
\text { bias }\end{array}$ & GRADE \\
\hline \multicolumn{12}{|l|}{ DKK-1 used alone } \\
\hline Overall & $31(52)$ & 11718 & $\begin{array}{l}0.70 \\
(0.69-0.71)\end{array}$ & $\begin{array}{l}0.82 \\
(0.81-0.83)\end{array}$ & $\begin{array}{l}4.59 \\
(3.50-6.01)\end{array}$ & $\begin{array}{l}0.34 \\
(0.29-0.39)\end{array}$ & $\begin{array}{l}14.49 \\
(9.72-21.60)\end{array}$ & 0.8365 & 0.06 & 0.893 & High \\
\hline HCC & $17(35)$ & 9080 & $\begin{array}{l}0.71 \\
(0.69-0.72)\end{array}$ & $\begin{array}{l}0.87 \\
(0.86-0.88)\end{array}$ & $\begin{array}{l}5.12 \\
(4.08-6.41)\end{array}$ & $\begin{array}{l}0.33 \\
(0.29-0.37)\end{array}$ & $\begin{array}{l}17.08 \\
(12.83-22.74)\end{array}$ & 0.8515 & & 0.208 & High \\
\hline $\begin{array}{l}\text { Compared with } \\
\mathrm{LC} \pm \mathrm{HBV} \pm \mathrm{HCV} \text { in } \\
\mathrm{HCC}\end{array}$ & $7(16)$ & 3964 & $\begin{array}{l}0.71 \\
(0.68-0.73)\end{array}$ & $\begin{array}{l}0.86 \\
(0.84-0.87)\end{array}$ & $\begin{array}{l}4.79 \\
(3.77-6.09)\end{array}$ & $\begin{array}{l}0.34 \\
(0.30-0.39)\end{array}$ & $\begin{array}{l}14.98 \\
(10.73-20.92)\end{array}$ & 0.8201 & 0.36 & 0.755 & High \\
\hline Early HCC & $5(11)$ & 2885 & $\begin{array}{l}0.73 \\
(0.70-0.76)\end{array}$ & $\begin{array}{l}0.90 \\
(0.88-0.91)\end{array}$ & $\begin{array}{l}6.17 \\
(4.16-9.16)\end{array}$ & $\begin{array}{l}0.31 \\
(0.28-0.35)\end{array}$ & $\begin{array}{l}22.14 \\
(13.96-35.09)\end{array}$ & 0.8258 & 0.61 & 0.688 & High \\
\hline PC & $6(9)$ & 1386 & $\begin{array}{l}0.72 \\
(0.69-0.75)\end{array}$ & $\begin{array}{l}0.73 \\
(0.70-0.76)\end{array}$ & $\begin{array}{l}4.16 \\
(2.15-8.04)\end{array}$ & $\begin{array}{l}0.23 \\
(0.12-0.45)\end{array}$ & $\begin{array}{l}19.78 \\
(5.35-73.08)\end{array}$ & 0.8818 & & 0.712 & Moderate \\
\hline Early PC & $1(2)$ & 154 & $\begin{array}{l}0.85 \\
(0.78-0.91)\end{array}$ & $\begin{array}{l}0.77 \\
(0.69-0.84)\end{array}$ & $\begin{array}{l}3.69 \\
(2.69-5.07)\end{array}$ & $\begin{array}{l}0.19 \\
(0.12-0.30)\end{array}$ & $\begin{array}{l}19.23 \\
(10.07-36.72)\end{array}$ & - & 0.28 & - & Low \\
\hline GC+EPC & $6(6)$ & 1079 & $\begin{array}{l}0.65 \\
(0.61-0.69)\end{array}$ & $\begin{array}{l}0.53 \\
(0.49-0.57)\end{array}$ & $\begin{array}{l}3.53 \\
(1.15-10.88)\end{array}$ & $\begin{array}{l}7.67 \\
(3.93-14.95)\end{array}$ & $0.09(0.04-0.19)$ & 0.7358 & & 0.289 & Moderate \\
\hline \multicolumn{12}{|c|}{ DKK-1 in combination with other tumour marker } \\
\hline DKK-1+AFP & & & & & & & & & & & \\
\hline HCC & $12(30)$ & 7683 & $\begin{array}{l}0.91 \\
(0.82-0.96)\end{array}$ & $\begin{array}{l}0.88 \\
(0.87-0.89)\end{array}$ & $\begin{array}{l}6.39 \\
(5.26-7.77)\end{array}$ & $\begin{array}{l}0.19 \\
(0.16-0.23)\end{array}$ & $\begin{array}{l}36.57 \\
(26.30-50.86)\end{array}$ & $0.9211^{*}$ & & 0.945 & High \\
\hline $\begin{array}{l}\text { Compared with } \\
\mathrm{LC} \pm \mathrm{HBV} \pm \mathrm{HCV} \text { in } \\
\mathrm{HCC}\end{array}$ & $5(12)$ & 2654 & $\begin{array}{l}0.80 \\
(0.78-0.82)\end{array}$ & $\begin{array}{l}0.82 \\
(0.79-0.84)\end{array}$ & $\begin{array}{l}4.36 \\
(3.69-5.17)\end{array}$ & $\begin{array}{l}0.23 \\
(0.17-0.29)\end{array}$ & $\begin{array}{l}20.26 \\
(14.22-28.87)\end{array}$ & 0.8876 & 0.09 & 0.582 & High \\
\hline Early HCC & $5(13)$ & 3138 & $\begin{array}{l}0.84 \\
(0.82-086)\end{array}$ & $\begin{array}{l}0.87 \\
(0.85-0.88)\end{array}$ & $\begin{array}{l}5.56 \\
(4.20-7.37)\end{array}$ & $\begin{array}{l}0.19 \\
(0.14-0.26)\end{array}$ & $\begin{array}{l}30.33 \\
(18.37-50.09)\end{array}$ & $0.9109^{*}$ & 0.47 & 0.524 & High \\
\hline \multicolumn{12}{|l|}{ DKK-1+CA19-9 } \\
\hline PC & $4(7)$ & 1109 & $\begin{array}{l}0.96 \\
(0.94-0.97)\end{array}$ & $\begin{array}{l}0.72 \\
(0.68-0.76)\end{array}$ & $\begin{array}{l}3.70 \\
(2.55-5.36)\end{array}$ & $\begin{array}{l}0.06 \\
(0.04-0.11)\end{array}$ & $\begin{array}{l}80.46 \\
(46.37-139.6)\end{array}$ & $0.9563^{*}$ & & 0.881 & High \\
\hline Early PC & $1(2)$ & 250 & $\begin{array}{l}0.98 \\
(0.94-1.00)\end{array}$ & $\begin{array}{l}0.67 \\
(0.58-0.75)\end{array}$ & $\begin{array}{l}2.92 \\
(2.26-3.76)\end{array}$ & $\begin{array}{l}0.02 \\
(0.01-0.09)\end{array}$ & $\begin{array}{l}134.55 \\
(31.18-580.67)\end{array}$ & 0.500 & 0.58 & - & Moderate \\
\hline
\end{tabular}

AEGI, adenocarcinoma of oesophagogastric junction; CRC, colorectal carcinoma; DKK-1, dickkopf-1; ELISA, enzyme-linked immunosorbent assay; EPC, oesophageal carcinoma; GIC, gastrointestinal carcinomas; HCC, hepatocellular carcinoma; IHC, immunohistochemistry; IHCC, intrahepatic cholangiocarcinoma; LC, liver carcinomas; PC, pancreatic carcinomas, *high diagnostic value. 
Table 3. Summary estimates for the histopathological stratification from subgroup analyses depending on cancer subtype and DKK-1 test method used for the prognosis of GIC.

\begin{tabular}{|c|c|c|c|c|c|c|c|c|}
\hline $\begin{array}{l}\text { Histopathological } \\
\text { stratification }\end{array}$ & Subgroup type & No. of studies & $\begin{array}{l}\text { No. of } \\
\text { participants }\end{array}$ & OR $(95 \%$ CI $)$ & $P, I^{2}$ & Meta-regression & $\begin{array}{l}\text { Publication } \\
\text { bias }\end{array}$ & GRADE \\
\hline \multirow{7}{*}{$\begin{array}{l}\text { Tumour size }(>5 \\
\mathrm{cm} \text { vs } \leq 5 \mathrm{~cm})\end{array}$} & Overall & 8 & 1070 & $1.12(0.83,1.52)$ & $0.317,14.4 \%$ & & 0.631 & Moderate \\
\hline & Cancer type & & & & & 0.749 & & \\
\hline & $\mathrm{LC}$ & 4 & 295 & $1.22(0.53,2.83)$ & $0.096,52.8 \%$ & & 0.092 & Low \\
\hline & $\mathrm{HCC}$ & 3 & 245 & $1.15(0.35,3.75)$ & $0.043,68.3 \%$ & & 0.204 & Low \\
\hline & IHCC & 1 & 50 & $1.41(0.44,4.55)$ & & & - & Very low \\
\hline & GC-EPC & 4 & 775 & $1.06(0.79,1.42)$ & $0.667,0.0 \% \#$ & - & 0.319 & Moderate \\
\hline & GC & 3 & 704 & $1.01(0.74,1.37)$ & $0.822,0.0 \% \#$ & & - & Moderate \\
\hline \multirow{12}{*}{$\begin{array}{l}\text { TNM stage (III-IV } \\
\text { vs I-II) }\end{array}$} & Overall & 17 & 2366 & $1.80(0.91,3.59)$ & $0.000,91.0 \%$ & & 0.990 & Moderate \\
\hline & Cancer type & & & & & 0.227 & & \\
\hline & LC & 3 & 218 & $2.24(0.75,6.70)$ & $0.125,52.0 \%$ & & 0.777 & Very low \\
\hline & $\mathrm{HCC}$ & 2 & 168 & $1.54(0.36,6.54)$ & $0.113,60.3 \%$ & & - & Very low \\
\hline & IHCC & 1 & 50 & $4.91(1.22,19.71)^{*}$ & - & & - & - \\
\hline & GC-EPC & 12 & 1793 & $1.56(0.65,3.75)$ & $0.000,93.3 \%$ & & 0.830 & Moderate \\
\hline & GC & 7 & 1367 & $1.54(0.40,5.99)$ & $0.000,95.9 \%$ & & 0.727 & Moderate \\
\hline & EPC & 4 & 426 & $1.64(0.56,4.77)$ & $0.001,81.4 \%$ & & 0.357 & Moderate \\
\hline & PC & 2 & 355 & $3.16(0.99,10.07)$ & - & & - & Very low \\
\hline & Test method & & & & & 0.609 & & \\
\hline & ELISA & 5 & 453 & $1.19(0.49,2.91)$ & $0.006,72.1 \%$ & & 0.556 & Moderate \\
\hline & $\mathrm{IHC}$ & 12 & 1913 & $2.15(0.89,5.21)$ & $0.000,93.1 \%$ & & 0.990 & Moderate \\
\hline \multirow{7}{*}{$\begin{array}{l}\text { Differentiation } \\
\text { grade (Poor vs } \\
\text { Well/moderate) }\end{array}$} & Overall & 11 & 1664 & $1.01(0.70,1.47)$ & $0.018,55.0 \%$ & & 0.900 & Moderate \\
\hline & Cancer type & & & & & & & \\
\hline & LC(IHCC) & 1 & 50 & $0.65(0.18,2.29)$ & - & & - & Very low \\
\hline & $\mathrm{PC}$ & 2 & 355 & $0.49(0.09,2.72)$ & - & & - & Very low \\
\hline & GC-EPC & 8 & 1259 & $1.17(0.75,1.82)$ & $0.023,59.1 \%$ & & 0.393 & Moderate \\
\hline & GC & 4 & 959 & $1.07(0.83,1.81)$ & $0.029,66.7 \%$ & & 0.653 & Moderate \\
\hline & EPC & 3 & 300 & $1.51(0.55,4.15)$ & $0.099,56.8 \%$ & & 0.912 & Moderate \\
\hline $\begin{array}{l}\text { Lymphatic } \\
\text { invasion } \\
\text { (Yes/No) }\end{array}$ & Overall (GC) & 3 & 464 & $0.54(0.17,1.74)$ & $0.003,89.13 \%$ & & - & Moderate \\
\hline \multirow{9}{*}{$\begin{array}{l}\text { Lymph node } \\
\text { metastasis } \\
(\text { Yes/No) }\end{array}$} & Overall & 13 & 1898 & $1.10(0.53,2.29)$ & $0.000,90.2 \%$ & 0.245 & 0.830 & Moderate \\
\hline & LC(IHCC) & 1 & 50 & $5.18(0.89,30.09)$ & - & & - & Very low \\
\hline & PC & 2 & 355 & $1.37(0.21,8.90)$ & - & & - & Very low \\
\hline & GC-EPC & 10 & 1493 & $0.94(0.38,2.33)$ & $0.000,91.9 \%$ & & 0.756 & Moderate \\
\hline & GC & 6 & 1239 & $0.91(0.24,3.52)$ & $0.000,95.0 \%$ & & 0.917 & Moderate \\
\hline & EPC & 3 & 254 & $0.89(0.23,3.44)$ & $0.004,87.1 \%$ & & 0.730 & Moderate \\
\hline & Test method & & & & & 0.789 & & \\
\hline & ELISA & 3 & 273 & $1.22(0.33,4.50)$ & $0.005,80.8 \%$ & & 0.410 & Moderate \\
\hline & IHC & 10 & 1625 & $1.07(0.44,2.61)$ & $0.000,92.0 \%$ & & 0.974 & Moderate \\
\hline \multirow{10}{*}{$\begin{array}{l}\text { Vascular invasion } \\
\text { (Yes/No) }\end{array}$} & Overall & 10 & 1677 & $0.88(0.31,2.53)$ & $0.000,91.4 \%$ & & 0.840 & Moderate \\
\hline & Cancer type & & & & & 0.143 & & \\
\hline & LC & 4 & 246 & $1.69(0.83,3.44)$ & $0.419,0.0 \%^{\#}$ & & 0.776 & Moderate \\
\hline & $\mathrm{HCC}$ & 3 & 198 & $1.43(0.68,3.01)$ & $0.686,0.0 \%^{\#}$ & & 0.073 & Moderate \\
\hline & IHCC & 1 & 48 & $8.29(0.85,3.01)$ & - & & - & Very low \\
\hline & GC & 5 & 1120 & $0.53(0.12,2.42)$ & $0.000,95.3 \%$ & & 0.516 & Moderate \\
\hline & PC & 1 & 311 & $0.65(0.28,1.50)$ & - & & & \\
\hline & Test method & & & & & 0.507 & & \\
\hline & ELISA & 2 & 121 & $1.67(0.73,3.83)$ & $0.804,0.0 \% \#$ & & - & Very low \\
\hline & IHC & 8 & 1556 & $0.72(0.24,2.19)$ & $0.000,92.2 \%$ & & 0.736 & Moderate \\
\hline \multirow{6}{*}{$\begin{array}{l}\text { Distant metastasis } \\
\text { (Yes/No) }\end{array}$} & Overall & 7 & 1427 & $0.79(0.28,2.24)$ & $0.000,85.2 \%$ & & 0.797 & Moderate \\
\hline & Cancer type & & & & & 0.584 & & \\
\hline & $\mathrm{HCC}$ & 1 & 75 & $0.98(0.28,3.44)$ & - & & - & Very low \\
\hline & PC & 1 & 311 & $0.45(0.18,1.14)$ & - & & & \\
\hline & GC-EPC & 5 & 1041 & $0.85(0.20,3.73)$ & $0.000,89.7 \%$ & & 0.808 & Moderate \\
\hline & GC & 4 & 961 & $0.59(0.11,3.18)$ & $0.000,90.8 \%$ & & 0.617 & Moderate \\
\hline
\end{tabular}

AEGJ, adenocarcinoma of oesophagogastric junction; CRC, colorectal carcinoma; DKK-1, dickkopf-1; ELISA, enzyme-linked immunosorbent assay; EPC, oesophageal carcinoma; GIC, gastrointestinal carcinomas; HCC, hepatocellular carcinoma; IHC, immunohistochemistry; IHCC, intrahepatic cholangiocarcinoma; LC, liver carcinomas; PC, pancreatic carcinomas, *Significant difference, "No heterogeneity

\section{Discussion}

This manuscript focused on the diagnostic accuracy and prognostic significance of the biomarker protein DKK-1 in GIC. First, we conclude that DKK-1 has a moderate diagnostic value for overall GIC, a moderate diagnostic accuracy value for HCC and PC, and a low diagnostic accuracy value for EPC+GC. In addition, for the combined diagnostic value of
DKK-1+AFP, a high diagnostic accuracy value could be determined in the HCC and early HCC groups. However, the diagnostic efficiency of DKK-1+CA19-9 was better than that of DKK-1 alone, with an AUC value above 0.95 . Second, for the prognosis meta-analysis of histopathological stratification, we found that significant results occasionally appeared in the IHCC group. Furthermore, we found that EPC ranked best for the histopathological stratification of 
the prognosis, but there was no significant difference in these consequences. All of the above results show that DKK-1 has diagnostic accuracy and prognostic significance in GIC.

Generally, the accuracy of the diagnostic value was higher in HCC and PC, while the significance of prognostic efficacy was ranked first in EPC, which do not match. We think there are two reasons for this; first, the mismatch between diagnostic and prognostic data in the included publications. For example, 17 articles evaluated the DKK-1 diagnostic data of HCC, 5 articles evaluated the DKK-1 diagnostic data of PC, 3 articles evaluated the DKK-1 prognostic data of $\mathrm{HCC}$, and 2 articles evaluated the DKK-1 prognostic data of PC. In addition, regarding the diagnostic efficacy of AFP in HCC and CA19-9 in PC, it can be said that DKK-1 improves these diagnostic capabilities (Figure 2). However, the combined efficacy was not analysed in prognostic data due to limited original research (Table 3). Therefore, for the combined prognosis data of HCC and PC, the included articles were too few to obtain clinical recommendation results.

Second, prognostic indicators of HCC and PC were relatively small, mainly due to the rapid development of HCC and PC, and few prognostic data can be provided. Moreover, there was a large difference between HCC and PC in the prognosis data from the included studies, so the data that can evaluate the prognosis indicators had clinical heterogeneity[68]; therefore, there may be no significant differences in the HCC group in our study. However, there are also data suggesting the role of DKK-1 overexpression in HCC development, which was consistent with the results provided by our predictive and diagnostic data. In addition, DKK-1 may have a role in the aggressiveness of pancreatic carcinoma cells, which could serve as a novel biomarker[69]. In addition, the progression of GC and EPC is slower, so the prognosis data could be merged in our meta-analysis. For the detection of DKK-1, the diagnostic test was used to detect the expression of DKK-1 in serum by ELISA. For the prognostic test, ELISA and IHC can both be utilized. Moreover, there were no significant differences between the effects of the two detection methods (Table 3, Figure 3).

Similar results could be found in previous incomplete publications for the diagnostic value of DKK-1[70-72], and the combined diagnostic value of DKK-1+AFP could also be proven[70-71]. Moreover, Younis YS' s research revealed that serum DKK-1 has higher sensitivity, specificity, and accuracy in early diagnosis of HCC than AFP[73]. The above evidence proved that there was a positive correlation among the invasion, aggressiveness and malignancy of tumours and the expression of DKK-1. The diagnosis and prognosis of DKK-1 are of great value. DKK- 1 is an antagonist of the $\mathrm{Wnt} / \beta$-catenin pathway[74], and when investigating the correlation with cancer-related genes, high DKK-1 protein expression was associated with Wnt/ $\beta$-catenin and its downstream signalling pathway. Therefore, DKK-1 could be used as a biomarker for predicting the progression of GIC[75-77].

There are also some limitations among our systematic review and network meta-analysis. First, as previously mentioned, the results from the inclusion of diagnostic and prognostic data do not match, but we can still explain the reasons for such results. Second, the types of publications included are all observational studies, and the quality of the GRADE score results is not high, which may have a certain impact on the quality of our meta-analysis. Third, the results of significant differences were not found in our prognostic data. Our study included more studies of gastric cancer, but previous studies found significant differences[78-79], proving that many clinical data are still needed for meta-analyses.

Our systematic review and meta-analyses found that DKK-1 alone provided modest diagnostic accuracy value for overall GIC, HCC and PC, whereas diagnostic accuracy were effective in EPC and GC. In addition, DKK-1+AFP provides high diagnostic accuracy value for HCC and early HCC. DKK-1+CA19-9 provides high diagnostic accuracy value for PC. Additionally, our results also suggest that DKK-1 expression is higher in front ranking EPCs in the network meta-analysis. All of the above results show that DKK-1 has good diagnostic accuracy, especially the combinations of DKK-1+AFP in HCC and DKK-1+CA19-9 in PC, whereas these had modest prognostic significance in GIC. Therefore, in clinical practice, we encourage patients suspected of HCC and PC to conduct DKK-1 detection to improve the diagnostic accuracy. Future head-to-head studies are warranted for DKK-1 expression in HCC and PC tissues to evaluate the prognostic value of DKK-1 in histopathological stratification to find the balance between diagnostic and prognostic data.

\section{Supplementary Material}

Supplementary Table S1 Search strategies; Table S2 Main characteristics of DKK-1 in GIC;Table S3 Quality assessment of included studies by QUADAS-2 scales; Table S4 Quality assessment of included studies by Critical Appraisal Skills Programme (CASP) scales; Table S5 Summary estimates for the results from subgroup analyses depending on cancer type, cancer stage and control type using AFP alone or in combination with DKK-1 for the diagnosis of HCC; 
Table S6 SUCRA score from network meta-analysis for TNM stage and lymph node metastasis. http://www.jcancer.org/v11p7091s1.pdf

\section{Acknowledgments}

This study was was supported by Undergraduate Innovation and Entrepreneurship Training Program (201810163158).

\section{Author Contributions}

Yingshi Zhang proposed the study. Xiaowen Jiang, Fuhai Hui, Xiaochun Qin, Yuting Wu, Haihan Liu, Jing Gao, Xiang $\mathrm{Li}$ and Yali $\mathrm{Xu}$ collected and analyzed the data. Xiaowen Jiang, Fuhai Hui and Yingshi Zhang wrote the manuscript. All authors discussed the results and contributed to this work.

\section{Competing Interests}

The authors have declared that no competing interest exists.

\section{References}

1. Siegel R L, Miller K D, Jemal A. Cancer statistics, 2019. CA: a cancer journal for clinicians. 2019; 69(1): 7-34

2. Nguyen R, Bae S D W, Zhou G, et al. Application of organoids in translational research of human diseases with a particular focus on gastrointestinal cancers. Biochimica et Biophysica Acta (BBA)-Reviews on Cancer. 2020; 188350.

3. Tzartzeva K, Obi J, Rich N E, et al. Surveillance imaging and alpha fetoprotein for early detection of hepatocellular carcinoma in patients with cirrhosis: a meta-analysis. Gastroenterology. 2018; 154(6): 1706-1718. e1

4. Miller K D, Nogueira L, Mariotto A B, et al. Cancer treatment and survivorship statistics, 2019. CA: a cancer journal for clinicians. 2019; 69(5): 363-385.

5. Lu S, Stein J E, Rimm D L, et al. Comparison of biomarker modalities for predicting response to PD-1/PD-L1 checkpoint blockade: a systematic review and meta-analysis. JAMA oncology. 2019; 5(8): 1195-1204.

6. Bregni G, Sciallero S, Sobrero A. The Amount of Evidence Needed to Support ERBB2 as a Biomarker for Resistance to EGFR Inhibitors in Metastatic Colorectal Cancer-In Reply. JAMA oncology. 2019; 5(10): 1512-1512.

7. Chen ZY, Du Y, Wang L, et al. MiR-543 promotes cell proliferation and metastasis of renal cell carcinoma by targeting Dickkopf 1 through the Wnt/ $\beta$ -catenin signaling pathway. J Cancer. 2018; 9(20):3660-3668.

8. Krishnamurthy N, Kurzrock R. Targeting the Wnt/beta-catenin pathway in cancer: Update on effectors and inhibitors. Cancer treatment reviews. 2018; 62: 50-60.

9. Ye C, Qi L, Li X, et al. Targeting the NAD+ salvage pathway suppresses APC mutation-driven colorectal cancer growth and Wnt $\beta$-catenin signaling via increasing Axin level. Cell Communication and Signaling. 2020; 18(1): 1-17.

10. Monzavi N, Zargar S J, Gheibi N, et al. Angiopoietin-like protein 8 (betatrophin) may inhibit hepatocellular carcinoma through suppressing of the Wnt signaling pathway. Iranian Journal of Basic Medical Sciences. 2019; 22(10): 1166-1171.

11. Lorente L. New prognostic biomarkers of mortality in patients undergoing liver transplantation for hepatocellular carcinoma. World journal of gastroenterology. 2018; 24(37): 4230.

12. Igbinigie E, Guo F, Jiang S W, et al. Dkk1 involvement and its potential as a biomarker in pancreatic ductal adenocarcinoma. Clinica Chimica Acta. 2019; 488: 226-234.

13. Moher D, Liberati A, Tetzlaff J, et al. Preferred reporting items for systematic reviews and meta-analyses: the PRISMA statement. BMJ 2009; 339: b2535. doi:10.1136/bmj.b2535

14. McInnes MDF, Moher D, Thombs BD, et al. the PRISMA-DTA Group.Preferred Reporting Items for a Systematic Review and Meta-analysis of Diagnostic Test Accuracy Studies: The PRISMA-DTA Statement. JAMA 2018;319:388-96.

15. ROSPERO, 2009. Centre for reviews and dissemination. Systematic Reviews: CRD's Guidance for Undertaking Reviews in Health Care (Internet). University of York, York, England. http://www.cdr.york.ac.uk/prospero.

16. Whiting PF, Rutjes AW, Westwood ME, et al; QUADAS-2 Group. QUADAS-2: a revised tool for the quality assessment of diagnostic accuracy studies. Ann Intern Med. 2011; 155(8): 529-536.

17. Stang, A. Critical evaluation of the Newcastle-Ottawa scale for the assessment of the quality of nonrandomized studies in meta-analyses. Eur J Epidemiol. 2010; 25: 603-5.
18. Rutter CM, Gatsonis CA. A hierarchical regression approach to meta-analysis of diagnostic test accuracy evaluations. Stat Med 2001; 20: 2865-84.

19. Fernando SM, Tran A, Cheng W, et al. Diagnosis of elevated intracranial pressure in critically ill adults: systematic review and meta-analysis. bmj, 2019;366: 14225 .

20. Higgins JPT, Thompson SG. Quantifying heterogeneity in a meta-analysis. Statistics in medicine. 2002; 21(11): 1539-1558.

21. Feng F, Jiang Q, Jia $H$, et al. Which is the best combination of TACE and Sorafenib for advanced hepatocellular carcinoma treatment? A systematic review and network meta-analysis. Pharmacological research. 2018; 135: 89-101.

22. Sonbol MB, Mountjoy LJ, Firwana B, et al. The Role of Maintenance Strategies in Metastatic Colorectal Cancer: A Systematic Review and Network Meta-analysis of Randomized Clinical Trials. JAMA oncology. 2019; e194489-e194489.

23. Brozek JL, Akl EA, Jaeschke R, et al. GRADE Working Group. Grading quality of evidence and strength of recommendations in clinical practice guidelines: Part 2 of 3 . The GRADE approach to grading quality of evidence about diagnostic tests and strategies. Allergy 2009; 64:1109-16.

24. Balshem $\mathrm{H}$, Helfand $\mathrm{M}$, Schünemann $\mathrm{H}$ J, et al. GRADE guidelines: 3 . Rating the quality of evidence. Journal of clinical epidemiology. 2011; 64(4): 401-406.

25. Hu W, Hou Y. Expression and clinical value of glycogen synthase kinase-3 $\beta$, Dickkopf-1 and $\beta$-catenin in proximal gastric cancer.Chin J Prim Med Pharm.2020; 27,68-72.

26. N Zekri AR, El Kassas M, Salam ESE, et al.The possible role of Dickkopf-1, Golgi protein- 73 and Midkine as predictors of hepatocarcinogenesis: a review and an Egyptian study.Sci Rep.2020;10: 5156.

27. Zheng, X.The effect of serum AFP, TK1 and DKK1 levels on the diagnosis of primary liver cancer. Laboratory Medicine.2019, 12

28. Chen ZR, Huang W, Chen LZ, et al. Diagnostic value of combined detection of serum $\alpha$-fetoprotein, thymidine kinase 1 and dickkopf WNT signaling pathway inhibitor 1 in primary liver cancer. ONCOLOGY PROGRESS. 2019; 17(17): 2059-2065.

29. Hassan NAM, Mohammed EF, Mahran ZG, et al. Evaluation of serum dickkopf-1 as a novel biomarker for hepatocellular carcinoma. Journal of Current Medical Research and Practice. 2019; 4(1): 91

30. Xi LT, Zhang HX, Wu AR, et al. Value of combined measurement of serum alpha-fetoprotein, Dickkopf-1, and cytoskeleton-associated protein 4 in diagnosis hepatocellular carcinoma. J Clin Hepatol. 2019; 35(6):1276-1279.

31. Gu L, Xiao J, Wen F. Correlation research on the clinical expressions of OPN, sLAG-3 and DKK-1 in the gastric cancer tissues with its clinical pathological features. Chin J Clin Health. 2018; 21(5):648-652.

32. Hong S A, Yoo S H, Lee H H, et al. Prognostic value of Dickkopf-1 and B-catenin expression in advanced gastric cancer. BMC cancer. 2018; 18(1): 506.

33. Chen SO, Bo WB. Diagnosis value of combined detection of CA199,CA242 and DKK 1 in pancreatic cancer. Int J Lab Med. 2017; 38(4):496-499.

34. Liu D J, Xie Y X, Liu X X, et al. The role of Dickkopf-1 as a potential prognostic marker in pancreatic ductal adenocarcinoma. Cell Cycle. 2017; 16(17): 1622-1629.

35. Qin QF, Weng J, Xu GX, et al. Combination of serum tumor markers dickkopf-1, DCP and AFP for the diagnosis of primary hepatocellular carcinoma. Asian Pacific journal of tropical medicine. 2017; 10(4): 409-413.

36. Xie HL, Chen F, Li L, et al. Clinical application of DKK-1, TFF3 and CA72-4 detection in diagnosis of gastric cancer. Int J Lab Med. 2017; 38(9): 1217-1219.

37. $\mathrm{Xu}$ YW, Guo $\mathrm{H}$, Guo HP, et al Serum level and clinical diagnostic value of DKK1 in adenocarcinoma at the esophago-gastric junction. Cancer Prevention Research. 2019; 29(2):139-142.

38. Yao F, Liu L, Zhang LA, et al. Diagnostic Value of Serum GP73 Combined with Dickkopf-1 and AFP in Hepatocellular Carcinoma. Progress in Modern Biomedicine. 2017; 17(26): 5153-5156.

39. Erdal H, Gül Utku Ö, Karatay E, et al. Combination of DKK1 and AFP improves diagnostic accuracy of hepatocellular carcinoma compared with either marker alone. Turk J Gastroenterol. 2016; 27(4): 375-81.

40. Fouad YM, Mohamed HI, Kamal EM, et al. Clinical significance and diagnostic value of serum dickkopf-1 in patients with hepatocellular carcinoma. Scandinavian journal of gastroenterology. 2016; 51(9): 1133-1137.

41. Mao LP, He YM, Han G, et al. Diagnosis Value of Detecting Plasma Dickkopf-1 Patients with Hepatocellular Carcinoma. J Mod Lab Med. 2016; 31(5): 62-65.

42. Su R, Su M, Lu Y. The diagnostic value of serum DKK1 in hepatocellular carcinoma. Shandong Medical Journal. 2016; 56(16): 75-76

43. Zhao PS, Weng RQ, Zhong ZX. Value of serum alpha-fetoprotein, Dickkopf 1 and interleukin-6 to diagnosis of hepatocellular carcinoma. J Clin Pract Diagn Ther. 2016; 30(8):780-782.

44. Fu Y, Wu HY, Gu JY, et al. Clinicopathological signifcance and correlation of C-MET, Golpll2, EZII2 and DKKl levels in human hepatocellular carcinoma. J Clin Exp Pathol. 2015; 31(12):1334-1338.

45. Ge T, Shen Q, Wang N, et al. Diagnostic values of alpha-fetoprotein, dickkopf-1, and osteopontin for hepatocellular carcinoma. Medical oncology. 2015; 32(3): 59

46. Han S, Zhou X, Sui X, et al. Serum dickkopf-1 is a novel serological biomarker for the diagnosis and prognosis of pancreatic cancer. Oncotarget. 2015; 6(23): 19907.

47. Ma G, Dai WJ, Yan W, et al. Expression of Dickkopf-1 in gastric cancer and its clinical significance. Clin J Dig. 2015; 35(12): 846-848. 
48. Ma X, Tan QW, Peng YF, et al. Prognostic significance of serum DKKl in hepatocellular carcinoma(HCC)patients. Fudan Univ J Med Sci. 2014; 41(5): 589-623.

49. Peng $\mathrm{YH}, \mathrm{Chen} \mathrm{JL}$, Weng XF, et al. Combined detection of serum DKK1 and P53 autoantibodies for the diagnosis value of esophageal squamous cell carcinoma. J Int Oncol.2015; 42(6):414-418.

50. Tan J. The Expression and Meaning of R-spondin2, R-spondin4 and Dickkopfl in Esophageal Cancer. Hebei Medical University. 2015.

51. Cai LY, Yin WJ, Xu MY. Clinical significance of ot serum and tissue Dickko pf-1 levels levels in patients with gastric cancer. China Journal of Modem Medicine. 2014; 24(6):1-8.

52. Wang QW, Wang QZ, Li DP, et al. Detection of Dkk-1 in pancreatic carcinoma and its clinical significance. J Bengbu Med Coll. 2014; 39(6): 718-723.

53. Zhou Y, Xu QH, Kang YP, et al. Detection and clinical significance of serum Dickkopf-1 in patient with pancreatic cancer. Shandong Medical Journal. 2014; 54(10):43-45.

54. Peng L.The values of Dickkopf-1(DKK1) in diagnosing primary carcinoma of the liver. Suzhou University. 2013.

55. Yang H, Chen GD, Fang F, et al. Dickkopf-1: as a diagnostic and prognostic serum marker for early hepatocellular carcinoma. The International journal of biological markers. 2013; 28(3): 286-297.

56. Gao C, Xie R, Ren C, et al. Dickkopf- 1 expression is a novel prognostic marker for gastric cancer. BioMed Research International. 2012; 2012.

57. Lee H S, Lee H E, Park D J, et al. Clinical significance of serum and tissue Dickkopf-1 levels in patients with gastric cancer. Clinica chimica acta. 2012; 413(21-22): 1753-1760.

58. Shen $\mathrm{Q}$, Fan J, Yang $\mathrm{XR}$, et al. Serum DKK1 as a protein biomarker for the diagnosis of hepatocellular carcinoma: a large-scale, multicentre study. The lancet oncology. 2012; 13(8): 817-826.

59. Zhang X, Shi RY, Yang XR, et al. Expression and Clinical Significance of Dickkopf-1 in Tissue and Serum Samples of Intrahepatic Cholangiocarcinoma. Chinese Journal of Clinical Medicine. 2012; 19(2): 102-104.

60. Zhang YJ, Jin XY, Liang DY. The Value of Serum Dickkopf I Protein for Early Diagnosis of Esophageal Cancer. Chin J ClinThoracCardiovasc Surg. 2012;19(6):659-662.

61. Soydinc H O, Duranyildiz D, Camlica H, et al. Lack of diagnostic potential of Dickkopf-1 in colon and rectum cancers. Asian Pac J Cancer Prev. 2011; 12(12): 3187-3189.

62. Tung EKK, Mak CKM, Fatima S, et al. Clinicopathological and prognostic significance of serum and tissue Dickkopf-1 levels in human hepatocellular carcinoma. Liver International. 2011; 31(10): 1494-1504.

63. Zhou Y, Wang D, Li ZS. Diagnostic Value of Dickkopf-1 in Endoscopic Ultrasound-guided Fine Needle Aspiration Specimen for Pancreatic Cancer. Gastroenterology. 2011; 16(1):7-10.

64. Zhang XG, Zhong L, Liu Q, et al. Expression and clinical significance of dkkl in gastric cancer tissue and serum. Shandong Medical Journal. 2010; 50(19):74-75.

65. Li BB.The express ion of serum DKK-1 in esophageal cancers and 1ts clinical research. Shandong academy of medical sciences. 2009.

66. Makino T, Yamasaki M, Takemasa I, et al. Dickkopf-1 expression as a marker for predicting clinical outcome in esophageal squamous cell carcinoma. Annals of surgical oncology. 2009; 16(7): 2058-2064.

67. Yamabuki T, Takano A, Hayama S, et al. Dikkopf-1 as a novel serologic and prognostic biomarker for lung and esophageal carcinomas. Cancer research. 2007; 67(6): 2517-2525.

68. Watany M, Badawi R, Elkhalawany W, et al. Study of Dickkopf-1 (DKK-1) gene expression in hepatocellular carcinoma patients. Journal of clinical and diagnostic research: JCDR. 2017; 11(2): OC32.

69. Takahashi N, Fukushima T, Yorita K, et al. Dickkopf-1 is overexpressed in human pancreatic ductal adenocarcinoma cells and is involved in invasive growth. International journal of cancer. 2010; 126(7): 1611-1620.

70. Zhang J, Zhao Y, Yang Q. Sensitivity and specificity of Dickkopf-1 protein in serum for diagnosing hepatocellular carcinoma: a meta-analysis. The International journal of biological markers. 2014; 29(4): 403-410.

71. Li Z, Mou L, Gao H, et al. Diagnostic accuracy of serum dickkopf-1 protein in diagnosis hepatocellular carcinoma: An updated meta-analysis. Medicine. 2019; 98(32).

72. Liang B, Zhong L, He Q, et al. Serum dickkopf-1 as a biomarker in screening gastrointestinal cancers: a systematic review and meta-analysis. OncoTargets and therapy. 2015; 8: 3115.

73. Younis YS, Alegaily HS, Elagawy W, et al. Serum Dickopff 1 as a Novel Biomarker in Hepatocellular Carcinoma Diagnosis and Follow Up After Ablative Therapy. Cancer Manag Res. 2019; 11: 10555-10562.

74. Cong LH, Du SY, Wu YN, et al. Upregulation of Klotho potentially inhibits pulmonary vascular remodeling by blocking the activation of the Wnt signaling pathway in rats with PM2.5-induced pulmonary arterial hypertension.J Cell Biochem. 2018;119 (7), 5581-5597.

75. Jaschke N, Hofbauer LC, Göbel A, et al. Evolving functions of Dickkopf-1 in cancer and immunity. Cancer Lett. 2020 Apr 3.

76. Wang $\mathrm{W}, \mathrm{He} \mathrm{Y}$, Rui J, et al. miR-410 acts as an oncogene in colorectal cancer cells by targeting dickkopf-related protein 1 via the $\mathrm{Wnt} / \beta$-catenin signaling pathway. Oncology letters. 2019;17(1): 807-814.

77. Li S, Liu F, Pei $\mathrm{Y}$, et al. Long noncoding RNA TTN-AS1 enhances the malignant characteristics of osteosarcoma by acting as a competing endogenous RNA on microRNA-376a thereby upregulating dickkopf-1. Aging (Albany NY). 2019;11:7678-7693.
78. Liu $\mathrm{Y}$, Tang $\mathrm{W}$, Xie $\mathrm{L}$, et al. Prognostic significance of dickkopf-1 overexpression in solid tumors: a meta-analysis. Tumor Biology. 2014; 35(4): 3145-3154.

79. Liu QR, Li YF, Deng ZQ, et al. Prognostic significance of Dickkopf-1 in gastric cancer survival: a meta-analysis. Genetic testing and molecular biomarkers. 2016; 20(4): 170-175. 